\section{Quanto custa para as famílias de baixa renda obterem uma dieta saudável no Brasil?}

\author{
The cost of meeting dietary guidelines for \\ low-income Brazilian families
}

\author{
¿Cuánto les cuesta una dieta saludable \\ a los brasileños de bajos ingresos?
}

\author{
${ }^{1}$ Faculdade de Saúde \\ Pública, Universidade de \\ Saúde Pública, São Paulo, \\ Brasil. \\ 2 Universidade Federal de \\ Minas Gerais, Belo Horizonte, \\ Brasil. \\ Correspondência \\ C. A. Borges \\ Faculdade de Saúde Pública, \\ Universidade de São Paulo. \\ Av. Dr. Arnaldo 715, São \\ Paulo, SP 01246-904, Brasil. \\ camilaborges.usp@gmail.com
}

\begin{abstract}
The objective was to identify the cost of meeting the Brazilian National Dietary Guidelines and analyze the impact on family budget. Data from the Brazilian Household Budget Survey for 2008 were used. Food purchases were recorded for seven days in 55,970 households. A subset of lowincome families $(\leq B R L 415.00$ per capita/month and $\leq U S \$ 1.00$ per capita/day) was used for the analysis. We estimated per capita calorie availability, total food expenditures, and food prices aggregated in 8 food groups based on the Brazilian Guidelines. Each food group's share in total calories was estimated and compared to the recommendations. Actual purchases exceeded the recommendations for beans, oils/fats, sweets, and meat/eggs, and fell short for fruits, vegetables, dairy products, and grains. Meeting the recommendations would increase food expenditures by 58\% among individuals with per capita income $\leq$ US\$ 1.00/day and by 39\% for those with per capita income $\leq B R L$ 415.00. Adoption of the recommendations would require $145 \%$ of total income. Meeting current recommendations would demand an increase in income or a policy to reduce food prices.
\end{abstract}

Health Food; Income; Feeding
Camila Aparecida Borges 1

Rafael Moreira Claro 2

Ana Paula Bortoletto Martins 1

Betzabeth Slater Villar 1

\section{Resumo}

O objetivo do estudo foi identificar o custo necessário para a obtenção de uma dieta saudável no Brasil e analisar o comprometimento dessa prática na renda familiar. Foram utilizados dados da Pesquisa de Orçamentos Familiares de 2008. Aquisições de alimentos foram coletadas durante sete dias em 55.970 domicílios. Dois subconjuntos compostos apenas por famílias de baixa renda $(\leq R \$ 415,00$ per capita/mês $e$ $\leq$ US\$1,00 per capita/dia) foram analisados. A partir dos alimentos obtidos, foram calculados calorias, despesa com alimentação e o preço médio dos oito grupos alimentares presentes no Guia Brasileiro. Foram comparados os gastos atuais e ideais para os oito grupos. As obtenções excederam às recomendações para feijões, óleos/ gorduras, doces, carnes/ovos e não alcançaram as recomendações para frutas, hortaliças, lácteos e cereais. Atingir as recomendações aumentaria os gastos com a alimentação em 58\%, para indivíduos de renda/per capita de $\leq U S \$ 1,00$ per capita/dia, e em 39\%, para indivíduos com renda $\leq R \$ 415,00$, e comprometeria em 145\% a renda familiar. Brasileiros de menor poder aquisitivo necessitam aumentar a renda para atingir a dieta ideal.

Alimentos Naturais; Renda; Alimentação 


\section{Introdução}

O estado de saúde dos indivíduos pertencentes às classes sociais mais baixas é inferior ao das classes sociais mais altas e está diretamente relacionado à qualidade inadequada da dieta 1,2 . No Brasil, o consumo alimentar das famílias de baixa renda é caracterizado pela grande presença de cereais, óleos e gorduras, açúcares, carnes gordas e alimentos industrializados, com alta densidade energética, em paralelo ao consumo insuficiente e monótono de hortaliças e frutas (praticamente restrito a tomate, alface, banana e laranja) 3 . Além disso, sabe-se ainda que, quando comparadas às famílias brasileiras de classes sociais mais altas, as famílias de menor nível de renda adquirem menos alimentos considerados saudáveis, como grãos integrais, peixes, leites e derivados desnatados, carnes magras e frutas e hortaliças 4 . O Guia Alimentar para a População Brasileira, versão 2006 , recomenda que os adultos sigam uma dieta balanceada de, aproximadamente, $2.000 \mathrm{kcal}$, com uma diversidade alimentar baseada na presença de, pelo menos, oito grupos alimentares que são: (i) Cereais, tubérculos, raízes e derivados; (ii) Feijões; (iii) Frutas e sucos de frutas naturais; (iv) Legumes e verduras; (v) Leites e derivados; (vi) Carnes e ovos; (vii) Óleos, gorduras e sementes oleaginosas; (viii) Açúcares e Doces 5 .

Escolhas alimentares são processos complexos, sendo influenciadas tanto por fatores biológicos quanto por fatores sociais, culturais e econômicos 6, com destaque, nesse último caso, para a renda dos indivíduos e o preço dos alimentos 4 . Especialmente no que concerne à ingestão de alimentos saudáveis, como grãos integrais, frutas e hortaliças, fatores econômicos parecem exercer influência decisiva 4,7. Na Austrália, por exemplo, a manutenção de uma dieta saudável representa um desafio para as famílias de baixa renda, dependentes de assistência social, visto que a compra de alimentos compromete cerca de $40 \%$ da renda total dessas famílias 2,8, e, no caso das famílias com renda média, o gasto com a compra de comida saudável compromete $20 \%$ do rendimento total 8 . Nesse país, seria necessário realocar até $1 / 3$ da renda semanal das famílias de baixa renda para que se alimentem de acordo com a recomendação nacional 8. Na Irlanda, uma família também de baixa renda, composta de dois adultos e duas crianças, precisaria gastar, aproximadamente, $69 \%$ da sua renda familiar semanal para alcançar a compra de uma cesta de alimentos considerada saudável nesse país ${ }^{9}$. Um outro estudo realizado na Itália concluiu que a adoção de uma dieta baseada nas recommended daily allowances (RDAs) acarretaria um aumento de, aproximadamente, um terço nos gastos atuais com a aquisição de alimentos pelos italianos 10 .

No Brasil, não se tem conhecimento de estudos sobre o custo de uma dieta nutricionalmente adequada (que atenda as recomendações do Guia Alimentar Para a População Brasileira, versão 2006) e seu impacto sobre o orçamento das famílias, especialmente aquelas de menor nível de renda. O objetivo deste estudo é identificar o custo necessário para a obtenção de uma dieta saudável no Brasil e analisar o comprometimento dessa prática no orçamento das famílias do país.

\section{Método}

\section{Amostragem e coleta dos dados}

Os dados utilizados para este estudo foram obtidos na Pesquisa de Orçamentos Familiares (POF), realizada pelo Instituto Brasileiro de Geografia e Estatística (IBGE) em 2008/2009, a qual correspondeu a uma amostra probabilística de 55.970 domicílios 11. A POF 2008/2009 utilizou plano amostral complexo (semelhante ao utilizado pela POF 2002/03), estratificado e conglomerado, com sorteio dos setores censitários em um primeiro estágio e de domicílios em um segundo. Para o sorteio, procedeu-se ao agrupamento dos 12.800 setores presentes no conjunto de setores censitário denominado "Amostra Mestra de Inquéritos Domiciliares" ou amostra comum. O número de setores sorteados em cada estrato foi proporcional ao número total de domicílios, adotando-se o critério de contemplar, pelo menos, três setores na amostra de cada estrato. Em seguida, em cada setor, foram sorteados os domicílios, por amostragem aleatória simples, sem reposição. O número de domicílios com entrevistas, por setor, foi fixado de acordo com a área da pesquisa (sendo 12 domicílios nos setores urbanos, 16 nos setores rurais). Os domicílios entrevistados dentro de cada estrato foram distribuídos uniformemente ao longo dos quatro trimestres de duração do estudo, de forma a reproduzir, em cada estrato, a variação sazonal de rendimentos e aquisições de alimentos (e de outros produtos) 11

Para este estudo, a população foi classificada em duas categorias: a primeira foi composta por famílias com renda per capita de até $\mathrm{R} \$$ 74,00 mensais (ou US\$ 1,00 diário, valor definido como linha de pobreza extrema pelo Banco Mundial) 12; a segunda foi composta por famílias com renda per capita de até $\mathrm{R} \$ 415,00$ mensais - valor de um salário mínimo em 15 de janeiro de 2009 (data referência da pesquisa) 11. O conjunto de domicílios estudado pela POF foi utili- 
zado para comparação com as duas categorias selecionadas. A renda média mensal per capita do primeiro grupo foi de $\mathrm{R} \$ 30,00$; no segundo, foi de $\mathrm{R} \$ 241,22$; enquanto que, para a população brasileira como um todo, foi de $\mathrm{R} \$ 899,49$ per capita. Para este estudo, a renda total per capita foi criada a partir das informações de rendimento monetário e não monetário, presentes na base de dados da POF 2008-2009 e que representam o conceito de rendimento bruto total. Sendo assim, a renda média mensal per capita é uma estimativa que corresponde ao somatório dos rendimentos brutos monetários dos componentes das unidades de consumo, exclusive os empregados domésticos e seus parentes, acrescido do total dos rendimentos não monetários das unidades de consumo 11 .

Foram utilizados os dados da aquisição de alimentos e bebidas destinados ao consumo domiciliar (adquiridos de forma monetária e obtidos de forma não monetária), coletados durante o período de 7 dias consecutivos em cada domicílio. Durante esse período, foram registradas diretamente informações detalhadas sobre cada produto adquirido: marca, quantidade, unidade, valor da despesa em Reais, local de compra, forma de obtenção, entre outros 11 .

Devido ao curto período de referência para a coleta de dados sobre aquisição de itens alimentares em cada domicílio, foram utilizados, como unidades de estudo, os registros de aquisições de itens de consumo de agregados de domicílios, denominados de estratos, que são geograficamente e socioeconomicamente homogêneos.

\section{Organização dos dados e criação das variáveis de estudo}

Os dados de alimentos para consumo domiciliar (adquiridos de forma monetária e obtidos de forma não monetária) geraram um total de 1.716 tipos de alimentos e bebidas que foram agrupados em oito grupos alimentares baseados no Guia Alimentar para a População Brasileira, versão 2006 5: (i) Cereais, tubérculos, raízes e derivados; (ii) Feijões; (iii) Frutas e sucos de frutas naturais; (iv) Legumes e verduras; (v) Leites e derivados; (vi) Carnes e ovos; (vii) Óleos, gorduras e sementes oleaginosas; (viii) Açúcares e doces. Aproximadamente, $6,7 \%$ da frequência total de alimentos encontrados na pesquisa não puderam ser classificados em nenhum dos oito grupos (p. ex.: temperos secos, condimentos e bebidas alcoólicas) e foram excluídos dessa análise. Porém, esses alimentos representaram, aproximadamente, $20 \mathrm{kcal} /$ per capita / dia, ou seja, menos do que $2 \%$ do total de calorias obtidas por meio de aquisições de alimentos de forma monetária e obtenção de alimentos de forma não monetária.

Foram obtidas as quantidades per capita diárias (em gramas) baseadas nos itens alimentares adquiridos para o consumo dentro do domicílio, obtidos de forma monetária e não monetária. A forma monetária de obtenção de alimentos inclui os seguintes itens: (1) monetária à vista para a unidade de consumo; (2) monetária à vista para outra unidade de consumo; (3) monetária a prazo para a unidade de consumo; e (4) monetária a prazo para outra unidade de consumo, e a forma de obtenção de alimentos não monetária inclui os seguintes aspectos: 1. doações; 2 . retirada do negócio; 3. trocas; 4. produção própria; 9. outra 11. As quantidades em gramas de todos os alimentos identificados na pesquisa, posteriormente, foram agrupadas de acordo com os oito grupos recomendados no Guia Alimentar para a População Brasileira, versão 2006.

Foi identificado o valor da despesa total para cada um dos oito grupos de alimentos. O valor, em reais, da despesa total paga por esses alimentos inclui a despesa monetária e não monetária. A despesa não monetária, de acordo com a publicação da POF 2008/2009 11, refere-se a valorações das despesas não monetárias realizadas pelos próprios informantes, considerando os preços vigentes no mercado local. Mais detalhes sobre os procedimentos de tratamento dos dados sobre despesas com alimentos e tratamento do efeito inflacionário sobre as informações de valores dos alimentos podem ser vistos na publicação realizada pelo IBGE 11 .

As quantidades de alimentos (em gramas) obtidos de forma monetária e não monetária foram usadas para simular a obtenção de uma cesta de alimentos composta pelos mesmos oito grupos de alimentos recomendados no Guia Alimentar para a População Brasileira, versão 2006. Também foi estimada a quantidade de calorias presentes em cada um dos oito grupos de alimentos da cesta. Foram utilizados, no texto, os termos despesa real, representando o valor gasto na obtenção dos oitos grupos de alimentos pelos domicílios, e despesa ideal, representando o valor gasto para atingir as recomendações do guia.

O preço médio de cada um dos oito grupos alimentares, expresso em $\mathrm{R} \$ / 1.000 \mathrm{kcal}$, foi obtido dividindo-se o total de despesas reais com o grupo de alimentos pelo total de calorias e multiplicando-se o resultado por mil.

\section{Análise dos dados}

A proporção de calorias provenientes de cada um dos oito grupos de alimentos sobre o total de calorias das aquisições foi estimada para o conjunto 
total da população estudada pela POF e segundo estratificações definidas de acordo com o nível de renda (população com renda $\leq$ US $\$ 1,00$ per capita/dia e renda $\leq \mathrm{R} \$ 450,00$ per capita/mês).

Em seguida, expandiram-se homogeneamente as quantidades reais de alimentos obtidos para consumo dentro do domicílio - encontrados na POF - de cada um dos grupos alimentares de modo a equiparar o total energético da cesta de alimentos obtida pelos domicílios ao total calórico proposto no Guia Alimentar Para a População Brasileira, versão 2006 (1.943kcal). Uma vez que esse tipo de ajuste não altera a participação dos grupos de alimentos dentro da cesta, ele permite comparar diretamente a cesta de alimentos obtida pelas famílias com aquela recomendação ideal, preconizada pelo Guia Alimentar para a População Brasileira. Para fazer esse ajuste, em primeiro lugar, foi calculada a razão entre as calorias totais propostas no guia e as calorias totais provenientes dos alimentos obtidos para consumo dentro dos domicílios. Em seguida, o valor gerado por essa razão (diferente para cada classe de renda) foi multiplicado pelas calorias per capita, transformando o valor calórico atual no valor calórico ajustado para cada um dos oito grupos alimentares.

Foram estimados o valor da despesa per capita diária real com cada grupo de alimentos $\mathrm{e}$ a despesa necessária para atender as recomendações do Guia Alimentar para a População Brasileira (despesa ideal) para as duas categorias de domicílios estudadas, bem como para o conjunto total da população. A fim de respeitar o componente hedônico do preço dos alimentos, a obtenção de uma cesta de alimentos ideal foi desenvolvida para cada estrato de renda estudado, utilizando os valores de preço originalmente pagos pelo próprio grupo populacional. Desse modo, variações na despesa com determinado grupo de alimentos representa exclusivamente variações da quantidade total adquirida e não variações na qualidade ou variedade das aquisições. Em seguida, os valores estimados para despesa diária per capita com alimentação foram somados (tanto as despesas reais quanto as despesas ideais) e multiplicados por 30 a fim de representar valores mensais de despesas. Finalmente, estimou-se a participação dos valores obtidos nos três cenários de renda. As análises foram realizadas em programa estatístico Stata 12 (Stata Corp., College Station, Estados Unidos), devido ao delineamento complexo da amostra da POF 2008/2009.

\section{Resultados}

A disponibilidade calórica total per capita média foi de $956.70 \mathrm{kcal}$ para a classe de renda inferior; de $1.277,74 \mathrm{kcal}$ para a classe de renda de até $\mathrm{R} \$$ $415,00 /$ per capita/mês e de $1.572,97 \mathrm{kcal}$ para a média de todos os domicílios brasileiros. A comparação da participação calórica dos grupos de alimentos de acordo com os estratos de renda expõe a maior obtenção de cereais, tubérculos, raízes e derivados entre as famílias de menor renda, que é cerca de $20 \%$ superior à participação calórica média desse grupo de alimentos na dieta brasileira. Por outro lado, é possível observar a elevação na participação calórica de produtos de origem animal (carnes e ovos, leites e derivados) e de frutas e hortaliças no total de calorias adquiridas com a elevação dos níveis de renda. Quando comparados aos valores preconizados pelo Guia Alimentar para a População Brasileira, evidencia-se que a participação calórica dos alimentos obtidos para consumo dentro do domicílio está acima do recomendado para feijões; carnes e ovos; óleos, gorduras e sementes oleaginosas; açúcares e doces e abaixo do recomendado para frutas e sucos de frutas; leite e derivados; legumes e verduras em todas as classes de rendimentos (Tabela 1).

A Tabela 2 apresenta os valores de preço por mil calorias dos oito grupos de alimentos nas três categorias de renda estudadas. Nota-se que o preço pago pela classe inferior de renda ( $<$ US $\$ 1,00$ / per capita) é menor para todos os grupos de alimentos quando comparado com as demais classes, com exceção dos legumes e verduras.

Observa-se uma discrepância entre as despesas reais e ideais que variaram segundo os grupos de alimentos e nível de renda dos indivíduos. Atender as recomendações do Guia Alimentar para a População Brasileira acarretaria um aumento de $58,1 \%$ nas despesas reais com alimentação (de $\mathrm{R} \$ 2,18$ per capita/dia para $\mathrm{R} \$ 3,47$ per capita/dia) para indivíduos com renda per capita menor que US\$ 1,00/dia; 39,6\% (de R\$2,63 per capita/dia para $\mathrm{R} \$ 3,67$ per capita/dia) para indivíduos com renda per capita de 1 salário mínimo (R\$ 415,00 em 2009 ) e $30,8 \%$ (de $\mathrm{R} \$ 3,19$ per capita/dia para $\mathrm{R} \$ 4,17$ per capita/dia) (Tabela 3 ).

A Figura 1 ilustra o padrão de despesa real e ideal com as cestas de alimentos estudado segundo classes de renda. Nota-se que o padrão de despesas foi semelhante entre os três grupos, sendo que as maiores despesas são na obtenção de carnes e ovos e cereais/tubérculos (Figura la). Para atingirem as recomendações, seria necessário aumentar as despesas com a obtenção de frutas e sucos de frutas, legumes e verduras e leite e derivados (Figura 1b).

Ao comparar o comprometimento da renda total per capita nos gastos com alimentação, observa-se que, para os domicílios situados na faixa de extrema pobreza (renda $\leq$ US $\$ 1,00$ / per capita) dia), o comprometimento da renda total com os 
Tabela 1

Participação relativa de cada grupo alimentar sobre as calorias totais provenientes dos alimentos obtidos para consumo dentro dos domićilios segundo as recomendações do Guia Alimentar para a População Brasileira e classes de renda. Brasil, 2008/2009

\begin{tabular}{|c|c|c|c|c|}
\hline Grupos de alimentos & $\begin{array}{c}\text { Guia } \\
\text { alimentar }\end{array}$ & $\begin{array}{l}\text { Domicílios com } \\
\text { renda até US\$1,00 } \\
\text { per capita/dia * }\end{array}$ & $\begin{array}{l}\text { Domicílios com } \\
\text { renda até } \mathrm{R} \$ 415,00 \\
\text { per capita/mês ** }\end{array}$ & $\begin{array}{l}\text { Total dos domicílios } \\
\text { brasileiros } * \star \star\end{array}$ \\
\hline $\begin{array}{l}\text { (i) Cereais, tubérculos, } \\
\text { raízes e derivados }\end{array}$ & 46,32 & 47,72 & 43,26 & 40,67 \\
\hline (ii) Feijões & 2,83 & 5,97 & 6,06 & 5,53 \\
\hline $\begin{array}{l}\text { (iii) Frutas e sucos de } \\
\text { frutas naturais }\end{array}$ & 10,81 & 2,04 & 1,83 & 2,55 \\
\hline (iv) Legumes e verduras & 2,32 & 0,52 & 0,81 & 1,03 \\
\hline (v) Leites e derivados & 18,53 & 3,31 & 5,11 & 6,05 \\
\hline (vi) Carnes e ovos & 9,78 & 10,73 & 12,89 & 13,89 \\
\hline $\begin{array}{l}\text { (vii) Óleos, gorduras e } \\
\text { sementes oleaginosas }\end{array}$ & 3,76 & 13,97 & 14,24 & 14,28 \\
\hline (viii) Açúcares e doces & 5,66 & 15,74 & 15,79 & 16,00 \\
\hline Total (\%) & 100,00 & 100,00 & 100,00 & 100,00 \\
\hline
\end{tabular}

* O valor de US\$1,00 per capita/dia foi convertido em Real de acordo com a cotação do Dólar em janeiro de 2009, sendo equivalente a $\mathrm{R} \$ 2,38 /$ dia ou a $\mathrm{R} \$ 71,4 /$ mês;

** A renda média para o total de domićlios brasileiros foi de $\mathrm{R} \$ 899,49$ per capita/mês;

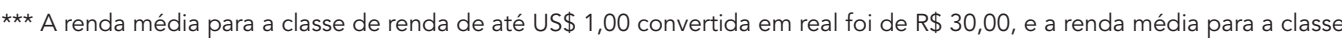
de renda de até $R \$ 415,00$ foi de $R \$ 241,22$.

Tabela 2

Preço por 1.000kcal dos oito grupos alimentares recomendados pelo Guia Alimentar para a População Brasileira segundo classes de renda. Brasil, 2008/2009.

\begin{tabular}{|c|c|c|c|}
\hline Grupos de alimentos & $\begin{array}{c}\text { Domicilios com renda } \\
\text { até US\$ } 1,00 \text { per } \\
\text { capita/dia * }\end{array}$ & $\begin{array}{c}\text { Domicilios com renda } \\
\text { até } \mathrm{R} \$ 415,00 \text { per } \\
\text { capita/mês ** }\end{array}$ & $\begin{array}{c}\text { Total dos } \\
\text { domicílios } \\
\text { brasileiros *** }\end{array}$ \\
\hline (i) Cereais, tubérculos, raízes e derivados & 0,77 & 0,88 & 1,00 \\
\hline (ii) Feijões & 0,84 & 0,91 & 0,92 \\
\hline (iii) Frutas e sucos de frutas naturais & 3,08 & 3,24 & 3,77 \\
\hline (iv) Legumes e verduras & 9,29 & 8,79 & 9,39 \\
\hline (v) Leites e derivados & 2,65 & 2,66 & 3,02 \\
\hline (vi) Carnes e ovos & 3,21 & 3,56 & 4,00 \\
\hline (vii) Óleos, gorduras e sementes & 0,45 & 0,46 & 0,56 \\
\hline \multicolumn{4}{|l|}{ oleaginosas } \\
\hline (viii) Açúcares e doces & 0,64 & 0,78 & 1,08 \\
\hline Total & 20,93 & 21,28 & 23,73 \\
\hline
\end{tabular}

* O valor de US\$1,00 per capita/dia foi convertido em Real de acordo com a cotação do Dólar em janeiro de 2009, sendo equivalente a $\mathrm{R} \$ 2,38 /$ dia ou a $\mathrm{R} \$ 71,4 /$ mês;

** A renda média para o total de domicílios brasileiros foi de $\mathrm{R} \$ 899,49$ per capita/mês;

*** A renda média para a classe de renda de até US\$1,00 convertida em real foi de $\mathrm{R} \$ 30,00$, e a renda média para a classe de renda de até $R \$ 415,00$ foi de $R \$ 241,22$. 
Tabela 3

Valores per capitas das despesas diárias reais e ideais para cada grupo de alimento estudado e a diferença (delta) entre eles, segundo as classes de renda. Brasil, 2008/2009

\begin{tabular}{|c|c|c|c|c|c|c|c|c|c|}
\hline \multirow[t]{2}{*}{ Grupos alimentares } & \multicolumn{3}{|c|}{$\begin{array}{l}\text { Domicílios com renda até } \\
\text { US\$ } 1,00 \text { per capita/dia * }\end{array}$} & \multicolumn{3}{|c|}{$\begin{array}{l}\text { Domicílios com renda até } \\
\text { R\$ } 415,00 \text { per capita/mês ** }\end{array}$} & \multicolumn{3}{|c|}{$\begin{array}{l}\text { Total dos domicílios } \\
\text { brasileiros *** }\end{array}$} \\
\hline & $\begin{array}{c}\text { Despesa } \\
\text { real }\end{array}$ & $\begin{array}{l}\text { Despesa } \\
\text { ideal }\end{array}$ & Delta & $\begin{array}{c}\text { Despesa } \\
\text { real }\end{array}$ & $\begin{array}{l}\text { Despesa } \\
\text { ideal }\end{array}$ & Delta & $\begin{array}{c}\text { Despesa } \\
\text { real }\end{array}$ & $\begin{array}{l}\text { Despesa } \\
\text { ideal }\end{array}$ & Delta \\
\hline (i) Cereais, tubérculos, raízes e derivados & 0,71 & 0,69 & $-0,02$ & 0,74 & 0,79 & 0,05 & 0,79 & 0,90 & 0,11 \\
\hline (ii) Feijões & 0,10 & 0,05 & $-0,05$ & 0,11 & 0,05 & $-0,06$ & 0,10 & 0,05 & $-0,05$ \\
\hline (iii) Frutas e sucos de frutas naturais & 0,12 & 0,65 & 0,53 & 0,11 & 0,68 & 0,56 & 0,19 & 0,79 & 0,60 \\
\hline (iv) Legumes e verduras & 0,09 & 0,42 & 0,33 & 0,14 & 0,40 & 0,26 & 0,19 & 0,42 & 0,23 \\
\hline (v) Leites e derivados & 0,17 & 0,95 & 0,78 & 0,27 & 0,96 & 0,70 & 0,35 & 1,09 & 0,74 \\
\hline (vi) Carnes e ovos & 0,67 & 0,61 & $-0,06$ & 0,89 & 0,68 & $-0,22$ & 1,08 & 0,76 & $-0,32$ \\
\hline $\begin{array}{l}\text { (vii) Óleos, gorduras e sementes } \\
\text { oleaginosas }\end{array}$ & 0,12 & 0,03 & $-0,09$ & 0,13 & 0,03 & $-0,09$ & 0,16 & 0,04 & $-0,12$ \\
\hline (viii) Açúcares e doces & 0,20 & 0,07 & $-0,13$ & 0,24 & 0,09 & $-0,15$ & 0,34 & 0,12 & $-0,22$ \\
\hline Total (R\$) & 2,18 & 3,47 & 1,29 & 2,63 & 3,67 & 1,03 & 3,19 & 4,17 & 0,97 \\
\hline
\end{tabular}

As despesas reais e ideais apresentadas na tabela levam, em consideração, os gastos relativos a uma dieta isoenergética 1.943kcal.

* O valor de US\$1,00 per capita/dia foi convertido em Real de acordo com a cotação do Dólar em janeiro de 2009, sendo equivalente a R\$2,38/dia ou

a $\mathrm{R} \$ 71,40 / \mathrm{mês}$;

** A renda média para o total de domicílios brasileiros foi de $\mathrm{R} \$ 899,49$ per capita/mês;

*** A renda média para a classe de renda de até US\$1,00 convertida em real foi de $R \$ 30,00$, e a renda média para a classe de renda de até $R \$ 415,00$

foi de $\mathrm{R} \$ 241,22$.

atuais gastos em alimentação é maior que $90 \%$, e, para atingir a despesa ideal em alimentação, o comprometimento seria de $145,8 \%$ (R\$ 104,10/ mês enquanto ganha $\mathrm{R} \$ 71,40$ em média). Por outro lado, para os domicílios com renda média mensal de $\mathrm{R} \$ 899,49$, a proporção do gasto com alimentação necessário para atingir as recomendações dietéticas é inferior ao atual comprometimento da renda com a alimentação $(4,52 \% v s$. 11,04\%) (Tabela 4).

\section{Discussão}

A obtenção de alimentos pelas famílias brasileiras sofre influência dos preços, como foi constatado a partir do registro das aquisições de alimentos realizado pela POF 2008/2009. Ao comparar os gastos atuais com alimentação no Brasil em diferentes estratos econômicos, observou-se que os domicílios em piores situações de renda (R\$ 71,40/mês) necessitariam aumentar sua despesa real com alimentos em $58,1 \%$, no intuito de atingir as recomendações nacionais de consumo, mesmo que optassem por obter alimentos para consumo que pertencessem aos mesmos grupos de alimentos obtidos atualmente. Tal incremento no gasto com a alimentação comprometeria a renda atual dessas famílias em $145,8 \%$, ou seja, nesse patamar de rendimentos, esses domicílios estariam impossibilitados de comprar a quantidade de alimentos ideal para o consumo. Identificou-se aquisição excessiva de óleos, gorduras e sementes oleaginosas; açúcares e doces; carnes e ovos em todas as situações estudadas, em paralelo a uma obtenção insuficiente de frutas e sucos de frutas; leite e derivados; cereais, tubérculos, raízes e derivados; legumes e verduras. As despesas alimentares com o grupo dos feijões se mostraram bem próximas das recomendações nutricionais. No entanto, observa-se uma alimentação desbalanceada em termos de variedade de grupos alimentares e energia. Esses achados evidenciam a impossibilidade de execução das recomendações de consumo do Guia Alimentar para a População Brasileira por parcela significativa da população brasileira $(23,3 \%$ da população recebia até $1 / 2$ salário mínimo e $27,2 \%$ de $1 / 2$ a 1 salário mínimo em 2009 - IBGE) 13.

No Brasil, este é o primeiro estudo que analisa os custos de se obter uma alimentação saudável, baseada nas recomendações nacionais propostas, em 2006, pelo Guia Alimentar para a População Brasileira, e que compara esses valores com os custos atuais praticados pela população, de acordo com as despesas reais em 
Figura 1

Padrão das despesas atuais e ideais com os grupos de alimentos estudados, segundo classes de renda. Brasil, 2008/2009.

1a) Despesas atuais

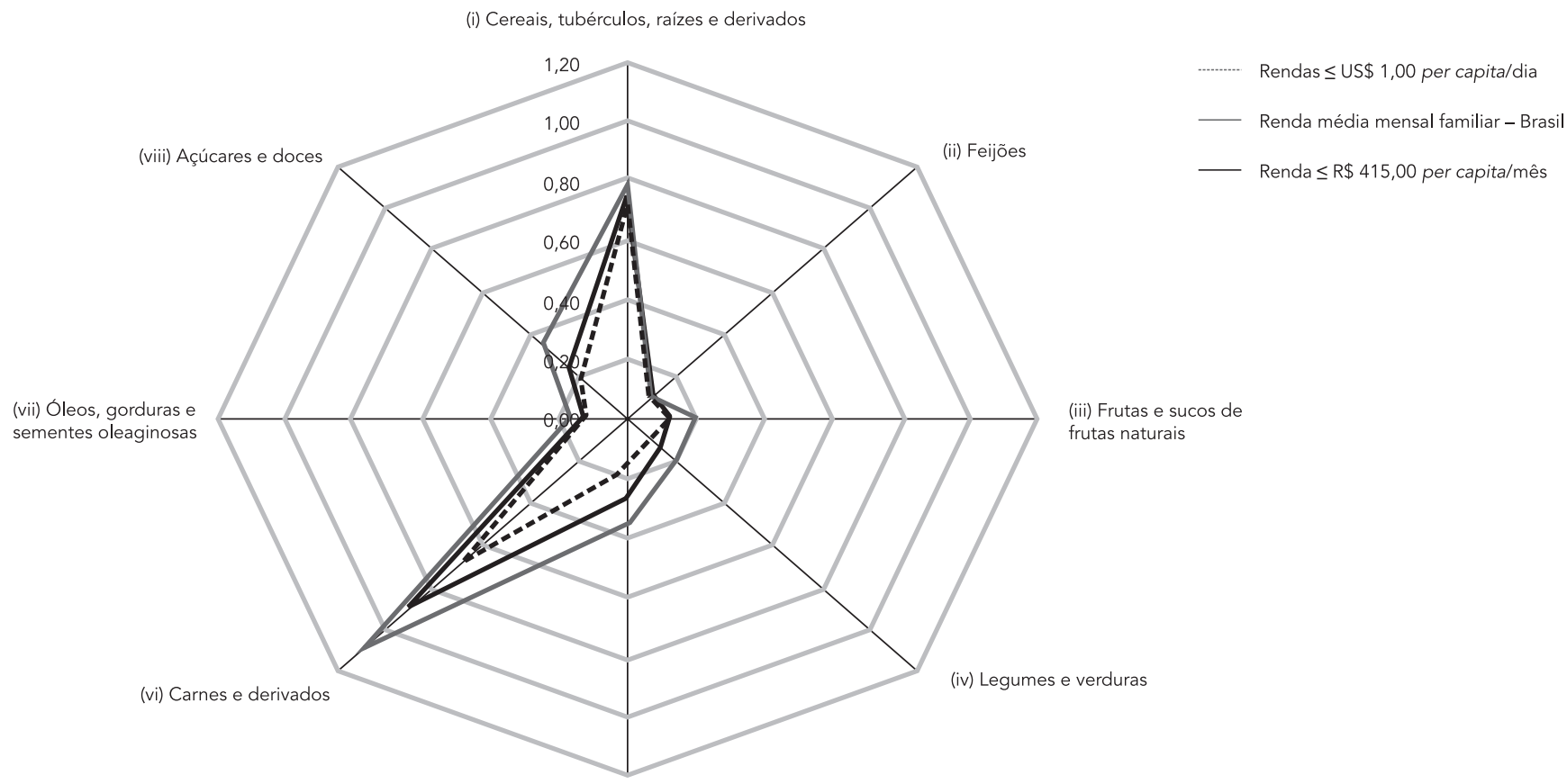

(v) Leites e derivados

1b) Despesas ideais

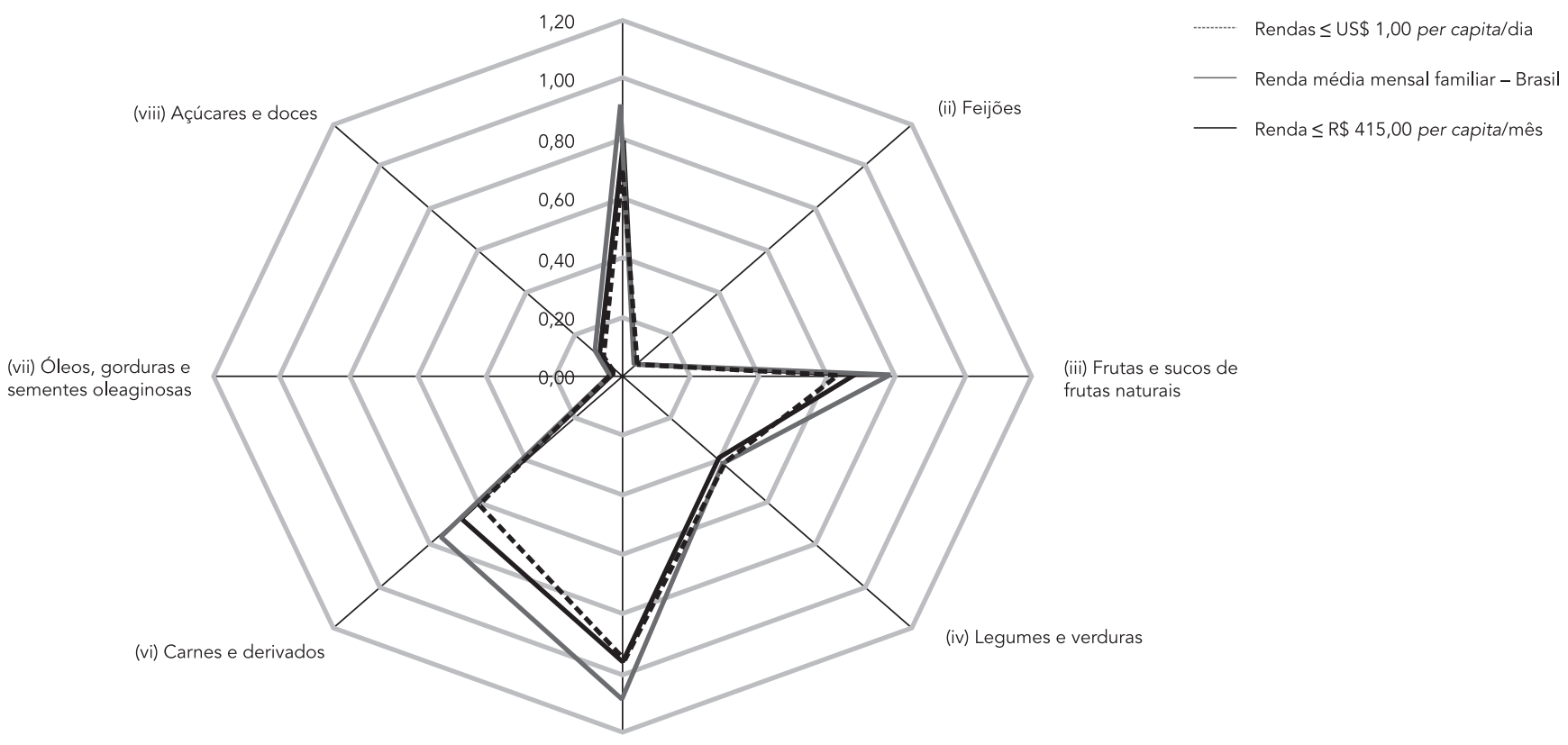

(v) Leites e derivados 
Tabela 4

Comprometimento da renda com as despesas reais em alimentação e com as despesas ideais (para atingir as recomendações nutricionais de energia do Guia Alimentar para a População Brasileira). Brasil, 2008/2009.

\begin{tabular}{|c|c|c|c|}
\hline Renda comprometida & $\begin{array}{l}\text { Domicílios com renda até US\$ } \\
1,00 \text { per capita/dia * }\end{array}$ & $\begin{array}{c}\text { Domicílios com renda até } \mathrm{R} \$ \\
415,00 \text { per capita/mês }\end{array}$ & $\begin{array}{c}\text { Total dos domicílios } \\
\text { brasileiros ** }\end{array}$ \\
\hline Despesa mensal ideal & $\mathrm{R} \$ 104,10$ & $\mathrm{R} \$ 110,10$ & $\mathrm{R} \$ 125,10$ \\
\hline Comprometimento da renda com despesa ideal & $145,80 \%$ & $26,53 \%$ & $4,52 \%$ \\
\hline Comprometimento da renda com despesa real & $91,60 \%$ & $19,01 \%$ & $11,04 \%$ \\
\hline
\end{tabular}

alimentação, praticadas pelos domicílios brasileiros. Para se colocarem em prática as recomendações dietéticas nacionais, uma série de desafios deve ser superada. As estratégias alimentares para a promoção de uma alimentação saudável precisa levar, em conta, os hábitos alimentares da população, a acessibilidade ao alimento, a conveniência, a variedade local de alimentos, os preços dos alimentos e, principalmente, os custos da dieta que parecem ser uma das principais barreiras para a manutenção de uma dieta saudável e para mudanças de comportamento alimentar em países desenvolvidos e em desenvolvimento 2,14,15

Nossos resultados acompanham as mudanças observadas no Brasil nas últimas 3 décadas. A participação dos principais grupos de bens e serviços nas despesas familiares se alterou consideravelmente desde os anos de 1970 11. As despesas com alimentação tiveram queda acentuada entre 1974/1975 (33,9\%) e 2002/2003 (20,8\%), mantendo a redução até 2008/2009 (19,8\%). Na área rural, as participações da alimentação nas despesas totais também foram se reduzindo, de $53,2 \%$ para $34,1 \%$, e, depois, para $27,6 \%$, respectivamente. Em contrapartida, nos últimos anos, a participação urbana da alimentação fora do domicílio nos gastos com alimentação subiu de $25,7 \%$ para $33,1 \%$, e a rural subiu de $13,1 \%$ para $17,5 \%$, evidenciando uma mudança de comportamento alimentar que nem sempre favorece escolhas saudáveis 11 .

Estudos nos Estados Unidos apontam que os preços dos alimentos representam uma barreira significativa para muitos consumidores que buscam alimentação adequada e com preços acessíveis 16. Por outro lado, estudo realizado por Drewnowski \& Eichelsdoerfer 17, também nos Estados Unidos, indicou que os preços dos alimentos em supermercados seguem o status econômico da região em que se encontra, ou seja, alimentos com altos preços em regiões com maior poder aquisitivo e alimentos com menores preços em áreas de menor poder socioeconômico, fato que reduz o efeito dos preços nas escolhas alimentares. As análises deste estudo levaram, em consideração, as diferenças de preços pagos por famílias de diferentes status de renda e também verificou o comprometimento da renda do brasileiro nas aquisições alimentares. Com isso, foi possível analisar que, no caso brasileiro, os preços dos alimentos ainda atuam como uma barreira para que as famílias de baixa renda façam escolhas alimentares inadequadas ou saudáveis.

Estudo realizado na Região Sul do Brasil com pré-escolares demonstrou uma alta prevalência de crianças que consumiam menos de uma porção de frutas e hortaliças por dia e que essas práticas estavam diretamente relacionadas com a educação dos pais e com a renda familiar 18 .

Outro estudo recentemente realizado nos Estados Unidos com o objetivo de identificar barreiras e facilidades no cumprimento das recomendações dietéticas americanas pela população de crianças rural e índioamericana encontrou que, dentre as barreiras, estão as preferências pessoais (p. ex.: "não gosto") e ambientais (p. ex.: "custa muito caro", "supermercado onde tem o produto disponível está muito longe de mim") 19 .

Os brasileiros de baixa renda gastam uma significativa parte da sua renda total em alimentos não saudáveis, com alta concentração de açúcares e gorduras. Porém, mesmo se esse dinheiro fosse realocado para aumentar a ingestão de frutas, hortaliças, leite e derivados, que são consumidos numa proporção muito menor atualmente, ainda assim não seria suficiente para adquirir uma dieta saudável. Assim, como foi verificado, para garantir um aporte adequado de frutas, hortaliças, leite e derivados em termos calóricos, seria necessário um aumento considerá- 
vel nos gastos com a alimentação, o que poderia ser superado com o aumento da renda dos indivíduos e ou a redução nos preços dos alimentos saudáveis.

Outros estudos também apontam que, quando os orçamentos familiares encolhem rapidamente, as pessoas mudam suas escolhas alimentares, adquirindo itens mais baratos e com alta densidade energética 20,21. Os primeiros itens que saem da dieta são geralmente os alimentos mais saudáveis, os quais a população não consegue alcançar as quantidades recomendadas, entre esses: grãos de alta qualidade de proteínas, hortaliças e frutas 2,22 . Os alimentos que se apresentam de baixo custo são, na maioria das vezes, alimentos ricos em energia, amidos, açúcares adicionados e gorduras vegetais, representando a forma mais barata de se alimentar 14 . As calorias provenientes de doces e gorduras são palatáveis, baratas, prontamente disponíveis e convenientes ao consumidor 22. Os alimentos frescos e nutritivos, muitas vezes, requerem habilidades culinárias, são mais caros e necessitam mais tempo para serem preparados 17 . Outro estudo sugere que a escolha por alimentos pobres em qualidade nutricional oferece satisfação ao paladar e reduz significativamente os custos da alimentação, por isso, esses alimentos são escolhidos no lugar de alimentos frescos, principalmente por famílias de baixa renda 2 .

Dentre os motivos que levam aos altos custos dos alimentos frescos e saudáveis, estão os grandes investimentos em armazenamento por parte dos produtores e comerciantes, os gastos com transporte e o maior desperdício por serem altamente perecíveis 20,23 . O contrário acontece com alimentos ricos em energia que são geralmente secos e tendem a ter maior tempo de prateleira 20 . Sendo assim, as calorias provenientes de alimentos com alta densidade energética são mais baratas do que as calorias provenientes de alimentos com alta concentração de nutrientes 20 .

Nosso estudo procurou observar as diferenças existentes entre as despesas reais com a alimentação das famílias brasileiras e as despesas ideais, seguindo uma dieta baseada em oito grupos alimentares recomendada pelo Guia Alimentar para a População Brasileira, versão 2006, que estabelece limites de consumo com base nos hábitos alimentares da população. Determinar o valor real das despesas com alimentação dentro e fora do domicílio pode contribuir para a elaboração de novas propostas alimentares projetadas para a população ou reestruturação das já existentes.

No Brasil, ainda não existe sistema de monitoramento dos gastos em alimentos que possa contribuir com a construção de planos alimentares para todas as camadas sociais. Em outros países como o Canadá, as despesas com alimentos são monitoradas, e é possível identificar exatamente o tipo e a quantidade de alimentos e bebidas adquiridos e os preços pagos pela população. Com os dados de compras disponíveis, é possível criar modelagens econômicas para identificar os reais custos de dietas saudáveis e propor ações de políticas públicas 24 .

Na Austrália, onde os custos da dieta recomendada são elevados e não são atingidos por famílias de baixa renda, foi identificada a importância de conhecer a porcentagem da renda comprometida com a alimentação, sobretudo nas famílias menos favorecidas 8 .

Apesar da falta de monitoramento dos preços, o governo federal anunciou a diminuição dos impostos sobre os alimentos da cesta básica e apresentou a reformulação dos itens presentes nela, inserindo outros alimentos de maior valor nutritivo 25. Em relação aos impostos, a desoneração inclui carnes (bovina, suína, aves, peixes, ovinos e caprinos), café, óleo, manteiga, açúcar, papel higiênico, pasta de dente e sabonete. Alimentos como leite, feijão, arroz, farinha de trigo ou massa, batata, legumes, pão e frutas, no momento, não sofrem tributação. Com essas medidas, será possível aumentar o poder de compra da população, movimentando, assim, a economia da população brasileira menos favorecida 25,26

Algumas limitações devem ser levadas em consideração aos achados desse estudo. Inicialmente, foram computados apenas os gastos com a obtenção de alimentos para o consumo dentro do domicílio, e, como citado anteriormente, houve um aumento nos gastos com a alimentação fora do domicílio nos últimos anos no Brasil 11 . Essa limitação pode ser explicada pelo fato de que a POF 2008/2009 não apresenta preços discriminados para cada item alimentar adquirido fora do domicílio. Uma vez que alimentos comprados fora do domicílio tendem a custar mais caros do que aqueles adquiridos para o consumo domiciliar (devido aos custos de preparação e distribuição, ausentes no consumo domiciliar), essa exclusão pode subestimar o custo de se atingir as recomendações do Guia Alimentar para a População Brasileira.

\section{Conclusão}

Identificou-se que acompanhar as indicações do Guia Alimentar para a População Brasileira implicaria num custo diário de R\$ 3,47, comprometendo cerca de $145,8 \%$ dos rendimentos da famílias com renda mensal de até $\mathrm{R} \$ 71,4$ e $26,53 \%$ das famílias com rendimento mensal de até $\mathrm{R} \$ 415,00$. Os brasileiros de menor poder 
aquisitivo necessitam aumentar suas despesas reais em alimentação para alcançarem uma dieta adequada, baseada nas recomendações nutricionais propostas pelo vigente Guia Alimentar para a População Brasileira, versão 2006. No entanto, para esse grupo de brasileiros, esse aumento na despesa com alimentação significaria comprometer praticamente toda a sua renda total, o que torna esse ato impossível de ser conduzido. Por outro lado, os brasileiros de outras camadas sociais destinam ou comprometem um considerável percentual de sua renda total na compra de alimentos pouco saudáveis. A quantidade da renda total destinada para a compra de alimentos tem um papel importante na aquisição de alimentos mais saudáveis por grande parte da população brasileira. Neste trabalho, foi verificada a impossibilidade de a classe social mais baixa da população manter uma dieta adequada seguindo propostas nacionais. Para reverter essa situação atual, podem ser destacados alguns caminhos que são facilitadores na busca de uma dieta mais saudável, entre eles, estão a importância de se ter uma política de redução de preços dos alimentos considerados mais saudáveis e a aplicação de impostos mais rigorosos sobre os alimentos com inferior qualidade nutricional. É necessário, também, que se adotem políticas como: incentivo à produção de alimentos locais ou regionais; educação alimentar, para a reorientação dos gastos com alimentos, que atualmente estão direcionados para a compra de alimentos pouco saudáveis; e uma melhor distribuição de renda, de forma a melhorar o poder aquisitivo da população. Todos esses fatores, agregados podem desempenhar um importante papel na promoção da alimentação saudável no Brasil e devem ser considerados eixos de discussão para a nova proposta do Guia Alimentar para a População Brasileira desenvolvido pelo Ministério da Saúde. Futuras análises como essa poderão ser realizadas, possibilitando estudos de comparações e mudanças ao longo tempo. Em longo prazo, recomenda-se a adoção de programas de monitoramento dos gastos em alimentação que contribuam para a melhor compreensão das barreiras econômicas que levam a hábitos alimentares inadequados.

\section{Resumen}

El objetivo de este estudio fue identificar los costes necesarios para mantener una dieta sana en Brasil y analizar la implicación de esta práctica en los ingresos. La metodología se basó en el análisis de datos de la Encuesta de Presupuestos Familiares 2008. Se recogieron las compras de alimentos durante siete días en 55.970 hogares. Se analizaron dos subconjuntos compuestos sólo por población con bajos ingresos (ingresos $\leq R \$$ 415,00 per cápita/mes e ingresos $\leq$ US\$1,00 per cápita/día). Se estimó la disponibilidad calórica/pc de las compras actuales, el gasto total en alimentos y el precio promedio de los ocho grupos de alimentos de la guía de alimentos de Brasil, además se realizó una compara- ción entre el gasto con lo recomendado. Las recomendaciones se excedieron en el caso de los frijoles, aceites/ grasas, dulces, carne/huevos, y no fueron las adecuadas en el caso de las frutas, verduras, lácteos y cereales. La consecución de las recomendaciones aumentaría el gasto en alimentos en un 58\%, en el caso de las personas cuyos ingresos/pc fueran $\leq$ US\$ 1,00 per cápita/día, y un $39 \%$ para las personas con ingresos $\leq R \$ 415,00$, comprometiendo un $145 \%$ de sus ingresos. Se debe analizar el desarrollo de una política de precios de los alimentos o aumentar los ingresos.

Alimentos Saludables; Renta; Alimentación 


\section{Colaboradores}

C. A. Borges e R. M. Claro contribuíram no desenho, análise, escrita e interpretação dos dados. A. P. B. Martins contribuiu na interpretação dos dados e revisão crítica do texto. B. Slater contribuiu na revisão crítica do texto. Todos os autores aprovaram a versão final do artigo.

\section{Agradecimentos}

Os autores agradecem à CAPES pelo financiamento de bolsa de estudo para este projeto.

\section{Referências}

1. Monteiro CA, Conde WL, Popkin BM. Income-specific trends in obesity in Brazil: 1975-2003. Am J Public Health 2007; 97:1808-12.

2. Drewnowski A, Darmon N. Food choices and diet costs: an economic analysis. J Nutr 2005; 135 : 900-4.

3. Coelho AB, Aguiar DRD, Fernandes EA. Padrão de consumo de alimentos no Brasil. Revista de Economia e Sociologia Rural 2009; 47:335-62.

4. Claro RM, Carmo HCE, Machado FMS, Monteiro CA. Renda, preço dos alimentos e participação de frutas e hortaliças na dieta. Rev Saúde Pública 2007; 41:557-64.

5. Ministério da Saúde. Guia alimentar para a população brasileira. Promovendo a alimentação saudável. http://189.28.128.100/nutricao/docs/geral/ guia_alimentar_conteudo.pdf (acessado em 26/ Jul/2012).

6. Frewer L, van Trijp H. Understanding consumers of food products. Cambridge: Woodhead Publishing; 2007.

7. Cade J, Upmeier H, Calvert C, Greenwood D. Costs of a healthy diet: analysis from the UK Women's Cohort Study. Public Health Nutr 1999; 2:505-12.

8. Kettings C, Sinclair AJ, Voevodin M. A healthy diet consistent with Australian health recommendations is too expensive for welfare-dependent families. Aust N Z J Public Health 2009; 33:566-72.
9. Friel S, Walsh O, McCarthy D. The irony of a rich country: issues of financial access to and availability of healthy food in the Republic of Ireland. J Epidemiol Community Health 2006; 60:1013-9.

10. Conforti P, D'Amicis A. What is the cost of a healthy diet in terms of achieving RDAs? Public Health Nutr 2000; 3:367-73.

11. Instituto Brasileiro de Geografia e Estatística. Pesquisa de Orçamentos Familiares 2008-2009. Aquisição alimentar per capita - Brasil e Grandes Regiões. http://www.ibge.com.br/home/estatistica/ populacao/condicaodevida/pof/2008_2009_aqui sicao/pof20082009_aquisicao.pdf (acessado em 10/Ago/2012).

12. Honohan P. Financial development, growth, and poverty: how close are the links? http://www.mi crofinancegateway.org/gm/document-1.9.26333/ 21113_wps3203.pdf (acessado em 21/Ago/2012).

13. Instituto Brasileiro de Geografia e Estatística. Séries estatísticas \& séries históricas. http://serieses tatisticas.ibge.gov.br/series.aspx?vcodigo=IU30\&s $\mathrm{v}=94 \& \mathrm{t}=$ rendimento-familiar-capita (acessado em 15/Jan/2013)

14. Drewnowski A, Darmon N, Briend A. Replacing fats and sweets with vegetables and fruits - a question of cost. Am J Public Health 2004; 94:1555-9. 
15. Masset G, Monsivais P, Maillot M, Darmon N Drewnowski A. Diet optimization methods can help translate dietary guidelines into a cancer prevention food plan. J Nutr 2009; 139:1541-8.

16. Center for Nutrition Policy and Promotion, U.S. Department of Agriculture. USDA food plans: cost of food report for December 2012. http://www. cnpp.usda.gov/ (acessado em 15/Jan/2013).

17. Drewnowski A, Eichelsdoerfer P. Can low-income americans afford a healthy diet? Nutr Today 2010; 44:246-9.

18. Valmórbida JL, Vitolo MR. Factors associated with low consumption of fruits and vegetables by preschoolers of low socio-economic level. J Pediatr (Rio J.) 2014; 90:152-60.

19. Jahns L, McDonald L, Wadsworth A, Morin C, Liu Y, Nicklas T. Barriers and facilitators to following the Dietary Guidelines for Americans reported by rural, Northern Plains American-Indian children. Public Health Nutr 2014; 28:1-8.

20. Darmon N, Briend A, Drewnowski A. Energy-dense diets are associated with lower diet costs: a community study of French adults. Public Health Nutr 2004; 7:21-8.

21. Andrieu E, Darmon N, Drewnowski A. Low-cost diets: more energy, fewer nutrients. Eur J Clin Nutr 2005; 60:434-6.

22. Drewnowski A, Darmon N. The economics of obesity: dietary energy density and energy cost. Am J Clin Nutr 2005; 82:265S-73.
23. Yeh M-C, Ickes SB, Lowenstein LM, Shuval K, Ammerman AS, Farris R, et al. Understanding barriers and facilitators of fruit and vegetable consumption among a diverse multi-ethnic population in the USA. Health Promot Int 2008; 23:42-51.

24. Ricciuto LE, Tarasuk VS. An examination of income-related disparities in the nutritional quality of food selections among Canadian households from 1986-2001. Soc Sci Med 1982 2007; 64:186-98.

25. Câmara dos Deputados. Medida Provisória no 609, de 8 de março de 2013. Reduz a zero as alíquotas da Contribuição para o PIS/PASEP, da COFINS, da Contribuição para o PIS/PASEP-Importação e da COFINS-Importação incidentes sobre a receita decorrente da venda no mercado interno e sobre a importação de produtos que compõem a cesta básica e dá outras providências. Diário Oficial da União 2013; 11 mar.

26. Nalon T. Governo zera impostos da cesta básica e muda sua composição. Folha de S. Paulo 2013. http://wwwl.folha.uol.com.br/mercado/ 1243366-governo-zera-impostos-da-cesta-basicae-muda-sua-composicao.shtml (acessado em 19/ Mar/2013).

Recebido em 16/Jan/2014

Versão final reapresentada em 05/Ago/2014

Aprovado em 08/Ago/2014 